\title{
EJES TRANSVERSALES Y PERFILES POR COMPETENCIA: UNA PROPUESTA VIABLE PARA SU EJECUCIÓN
}

\author{
llya Casanova Romero* \\ https://orcid.org/0000-0003-1147-7413 \\ Ítala Paredes Chacín** \\ https://orcid.org/0000-0003-0112-9212 \\ Engels Ortega Acurero*** \\ https://orcid.org/0000-0002-7389-6288
}

RECIBIDO: Enero 2020 / ACEPTADO: Mayo 2020 / PUBLICADO: Septiembre 2020

\begin{abstract}
Como citar: Casanova Romero, llya; Paredes Chacín, Ítala; Ortega Acurero, Engels. (2020). Ejes transversales y perfiles por competencia: una propuesta viable para su ejecución. Telos: revista de Estudios Interdisciplinarios en Ciencias Sociales, 22 (3), Venezuela. (Pp.510-527).

DOI: www.doi.org/10.36390/telos223.03
\end{abstract}

\section{RESUMEN}

El presente trabajo reconoce el potencial de la formación integral, la transversalidad, los currículos por competencias, la educación apoyada en el principio de interdisciplinariedad y el respeto a las disciplinas. Identifica como problemática, la falta de articulación de los elementos de la estructura curricular en los Planes de Estudio y sobre todo la ausencia de lineamientos que guíen el proceso de integración de los niveles curriculares, previsiones estas que deben estar prescritas en el nivel mesocurricular. El objetivo del trabajo fue proponer un núcleo procedimental sobre la base del modelo de integración curricular de los perfiles académicos-profesionales bajo un enfoque por competencias a partir de un ordenamiento transversal con la finalidad de superar el ordenamiento lineal tradicional. Se asumió un enfoque racionalista adoptándose el método deductivo, se construye y explica una estructura operativa denominada núcleo procedimental como soporte operativo al modelo teórico integración curricular del perfil por competencias a partir del ordenamiento transversal de Casanova (2018). El núcleo procedimental elaborado a

\footnotetext{
* PhD y Postdoctorado en Ciencias Humanas, Magíster en Educación Mención Planificación Educativa, Licenciada en Bioanálisis. Docente a Tiempo Completo de la Facultad de Ciencias Médicas de la Universidad Laica Eloy Alfaro de Manabí. Experiencia docente en pregrado y postgrado (especialidad, maestría y doctorado). Autora de capítulos de libro y artículos en revistas arbitradas de alto impacto nacionales e internacionales. Manta, Ecuador. Correo electrónico: ilya.casanova@uleam.edu.ec https://scholar.google.es/citations?user=GucTBXUAAAAJ\&hl=es\&safe=strict

** PhD en Ciencias Humanas, Magíster en Educación Mención Planificación Educativa, Licenciada en Educación Mención Ciencias Pedagogías Área Tecnología Instruccional. Experiencia docente en pregrado y postgrado a nivel internacional. Docente del Instituto de Posgrado de la Universidad Técnica del Norte Ecuador. Autora de capítulos de libro y artículos en revistas arbitradas de alto impacto. Ibarra, Ecuador. Correo electrónico: italmary76@gmail.com https://scholar.google.com.ec/citations?user=QKwqGacAAAAJ\&hl=es\&safe=strict

*** Magíster en Informática Educativa. Licenciado en Diseño Gráfico. Experiencia docente en pregrado y maestría. Secretario Docente de la Facultad de Arquitectura y Diseño de la Universidad del Zulia. Asesor y diseñador de revistas científicas y libros académicos y páginas Web institucionales. Maracaibo, Venezuela. Correo electrónico: engelsortega@gmail.com
} 
partir del modelo teórico cuenta de 7 (siete) fases, que de manera secuencial recorren los niveles curriculares para finalmente retornar al nivel mesocurricular donde se realiza la concreción definitiva de cada eje transversal, este procedimiento permite a los encargados de la gestión el seguimiento de lo declarado en sus perfiles profesionales. Se puede afirmar que la transversalidad como componente del currículo permite su organización y coherencia; al mismo tiempo que ofrece los mecanismos operativos para recorrerlo tanto en forma diacrónica como sincrónica. Adicional a esto, a través de la transversalidad se profundiza y consolidan todas las fases del desarrollo curricular además que se fortalece la formación integral, al proyectar el trabajo curricular en sus diversos niveles de concreción y facilitar el desarrollo de competencias académico-profesionales, tomando previsiones para la evaluación de sus indicadores de logro.

Palabras claves: currículo integral; competencias; transversalidad; formación integral; ordenamiento transversal.

\title{
Cross-cutting axes and profiles by competence: a viable proposal for its execution
}

\begin{abstract}
The present work recognizes the potential of integral education, transversality, curricula for competences, education supported by the principle of interdisciplinarity and respect for disciplines. Identifies as problematic, the lack of articulation of the elements of the curricular structure in the Study Plans and especially the absence of guidelines that guide the process of integration of the curricular levels forecasts that must be prescribed at the mesocurricular level. The objective of the work was to propose a procedural core based on the model of curricular integration of the academic-professional profiles under a competency-based approach based on a transversal order to overcome the traditional linear ordering. A rationalist approach was adopted adopting the deductive method, an operative structure called procedural core is constructed and explained as operative support to the theoretical model curricular integration of the profile by competences from a transversal order of Casanova (2018). The procedural core developed from the theoretical model has 7 (seven) phases, which sequentially cross the curricular levels to finally return to the mesocurricular level where the definitive concretion of each transverse axis is made, this procedure allows those in charge of the management monitoring of what was stated in their professional profiles. It can be affirmed that transversality as a component of the curriculum allows its organization and coherence; while it offers the operative mechanisms to cross it both diachronically and synchronously. In addition to this, through transversality, all the phases of curricular development are deepened and consolidated, as well as the integral formation is strengthened, by projecting the curricular work in its different levels of concretion and facilitating the development of academic-professional competences, taking forecasts for the evaluation of its achievement indicators.
\end{abstract}

Keywords: integral curriculum; competencies; transversality; integral formation; Cross-cutting organization. 


\section{Introducción}

Las transformaciones de los procesos de formación son necesarias y deben ser vistos de forma natural, en la actualidad, se requiere que la formación a nivel de educación superior plantee diseños curriculares más flexibles donde se admitan cambios a nivel operativo y se permita acercar lo formal escrito, a lo real ejecutado.

Si se concibe que el currículo tiene una dimensión de gestión muy importante para su desarrollo, en el mismo, deben exponerse los criterios epistemológicos, psicológicos, pedagógicos, de evaluación, de organización académica, comunicativos y administrativos, para justificar lo que el currículo selecciona, crea, organiza, transmite y produce en el diseño y desarrollo de contenidos, metodologías y lenguajes para los programas.

Abordar la elaboración de un diseño curricular supone, entre otras cosas, traducir los principios en normas, en prescripciones educativas, con la finalidad de elaborar un instrumento útil además de eficaz para la práctica pedagógica. El currículo es eslabón y centro, donde se sitúa la declaración de principios generales, su traducción operacional, entre teoría-práctica pedagógica, entre planificación-acción, entre lo establecido y lo que realmente sucede en el aula. Es lógico que la elaboración del currículo ocupe un lugar central en los planes de reforma educativa y se toma a menudo como punto de referencia para guiar otras actuaciones que aseguren la coherencia de las mismas (Coll, 2001).

En este punto se coincide con Vílchez (2004, pág. 201) quien define el currículo como "el conjunto de aprendizajes compartidos que la escuela, deliberada y espontáneamente, pone a disposición de estudiantes y maestros para que desarrollen plenamente sus potencialidades y participen en el proceso constante de transformación vital." Para Vílchez (2004) también es importante considerar en el diseño el plan de enseñanza y la atmósfera escolar, lo cual se complementa con lo señalado Bustamante et al (2007) quienes hacen referencia a la necesidad de implementar un currículo donde se favorezca el traslado de la concepción de la educación para el desarrollo integral de la persona y el principio de igualdad de oportunidades educativas.

El cambio educativo, compromete a la organización a un proceso de renovación, el cual parte del reconocimiento e institucionalización de las estrategias de mejora continua, donde se favorezcan y propicien escenarios con un clima de colaboración donde se discutan de forma crítica los mecanismos de resolución de conflictos.

Indudablemente, hoy es reconocido que no existe cambio sin involucrar a los actores del proceso, sin embargo, se requieren lineamientos medulares donde se contemplen diferentes escenarios en correspondencia con las políticas tanto gubernamentales como de la propia institución, lo cual garantizaría la transformación hacia estándares de calidad, eficiencia y productividad que, en el caso de las instituciones de educación superior, son cada vez más demandadas.

En la generalidad de los procesos de transformación curricular a nivel de educación superior, la mayor preocupación ha estado enfocada a la definición de los elementos conceptuales en los proyectos, sin que se definan lineamientos orientadores claros que faciliten el desarrollo coherente de otros elementos de la estructura como son el meso y el microcurrículo. En este sentido Horruitiner $(2006$, p. 1) refiere de acuerdo con su experiencia en diversas universidades latinoamericanas como asesor en procesos de diseño curricular 
(...) estos procesos se limitan a reformular los programas de estudio de las diferentes asignaturas y a debatir las horas lectivas que cada una de ellas requiere, sin consideraciones de carácter más general que permitan comprender con claridad cuál es el papel y el lugar de cada una de las materias de estudio y actuar en consecuencia con ello.

Para la gestión es indispensable unificar criterios en cuanto a las definiciones que manejaran los actores educativos en la institución. Para ello se iniciará considerando que existen tres planos perceptibles dentro de un proceso educativo tal como lo plantea Peñaloza (2005), los mismos son: (a) la concepción de la educación (fines), (b) el currículo y (c) los diseños de los componentes del currículo.

Bajo la concepción anterior, el currículo tendrá en su concreción tres (3) niveles: (a) nivel macro: donde se expresa de forma prescriptiva la concepción de la educación y propósitos cuya expresión resumida será el perfil académico-profesional (b) nivel meso: representado por la malla curricular, explicitándose el alcance de las áreas, líneas y ejes transversales, así como las previsiones de gestión curricular y (c) el nivel micro: el plano de los programas de las unidades curriculares (Casanova, 2011).

Desde esta visualización, el currículo se considera como la selección y previsión de los procesos y experiencias que deben vivir los educandos, siendo este el medio que permite la primera concreción en la realidad de la concepción de la educación. Es por ello por lo que se hace necesario evitar planificar de acuerdo a circunstancias coyunturales y así como la conformación de comisiones transitorias que solo pueden solventar los nudos críticos emanados de la operatividad del currículo; estas comisiones, en muchos casos no cuentan con los recursos humanos idóneos al no poseer la visión global y conocimiento necesarios para lograr el engranaje perfecto que permita el logro de los objetivos planteados.

Al respecto se hace pertinente lo planteado por Horruitiner (2006), donde alerta sobre la necesidad de iniciar todo proceso de transformación curricular con la preparación de quienes van a elaborar los nuevos currículos, en ideas, conceptos y principios que los sustentan. En el plano estratégico, tan importante como eso, es preparar a los profesores que van a aplicar esos currículos en los nuevos conceptos. Si el currículo llega a los profesores que han de aplicarlo como resultado de la elaboración teórica y aislada de un grupo selecto de expertos, entonces cada uno de los profesores lo adecuará a sus propias vivencias, en correspondencia con su formación pedagógica y profesional, y el resultado seguirá siendo insatisfactorio.

El currículo indiscutiblemente es una estructura compleja y es de esperar que ocurran desestabilizaciones, si es evaluado de manera permanente, las debilidades pueden ser vistas como oportunidades que permitirán reorientar el proceso de formación. Los problemas con frecuencia se solapan en lo operativo y tienen su génesis en procesos de gestión ineficaces, lo cual conduce a inconsistencias en el sistema curricular (Casanova, 2018).

Es importante enfatizar que el modelo que aquí se presenta asume lo señalado por Paredes (2011) quien refiere la importancia de asumir la construcción de un currículo basado en el enfoque por competencias, ya que el mismo representa una gran oportunidad para el cambio con apertura al mundo, a la sociedad del conocimiento y la pertinencia social de la formación profesional. En este sentido Paredes (2011, p.160), las competencias deben ser entendidas como: 
...procesos complejos de desempeños integrales para la resolución de problemas que implican conocimientos, idoneidad y ética, buscando la realización personal del hombre y su trascendencia, la calidad de todos los procesos vividos, el desarrollo social y sostenible en equilibrio con el contexto. En tal sentido, las competencias van más allá de los desempeños profesionales y de la acumulación de conocimientos disciplinares fragmentados.

Indudablemente, la configuración social, originada en los cambios de la circulación de los saberes, recrea un tipo de competencias sociales, culturales y cognitivas que no parecen apuntar en el sentido de las competencias para la generación de rentabilidad, capital y competitividad, predominante en el campo educativo, esto profundiza la brecha entre los intereses institucionales y la de los sujetos aprendices. La superación de esta situación pasa, entre otras cuestiones, por la incorporación de la transversalidad que rompa con el prejuicio que separa a las ciencias de las humanidades y por rescatar aquel tipo de saberes que sin tener una aplicación directa en la profesión son, sin embargo, socialmente útiles, los saberes lógicosimbólicos, históricos y estéticos (Martín-Barbero, 2003).

Es por ello por lo que se debe reforzar en la educación el principio de interdisciplinariedad donde se concilien los saberes que han permanecido fragmentados; desde esta perspectiva tendrá sustento la vinculación, cooperación y transferencia de las diferentes asignaturas, temáticas, competencias y logros científico-técnicos que se gestan en el mundo contemporáneo y repercuten en los sistemas de formación. Es importante la contribución al desarrollo sostenible y sustentable, a los ideales democráticos y a la convivencia solidaria entre las personas, las naciones, los pueblos y las culturas. (Casanova, 2018)

En este contexto tiene cabida la transversalidad vista como proceso, ella permite el desarrollo de un modelo de educación global, una nueva forma de concebir la práctica educativa que redefine el pensamiento que fundamenta el currículo y su praxis, a partir del reconocimiento de sus implicaciones complejas, sistémicas e interdisciplinarias, es una tecnología educativa generante, fomenta la integración a los problemas del entorno, parte de los conocimientos y experiencias previas, se expresa en el diseño, administración y evaluación educativa. (Bravo, 2006).

La transversalidad implica concienciación, encuentro, crítica reflexiva donde se evidencien las raíces ontológicas, se relacionen las disciplinas del saber al mismo tiempo que se integra el sentir de la sociedad donde está inmerso el ser. Es así como a partir de la transversalidad se persigue la integración de competencias relacionadas con lo que el alumno debe saber (lo cognoscitivo), saber hacer, saber emprender (lo procedimental), saber ser y convivir (lo actitudinal). La interdisciplinariedad desde la perspectiva de la transversalidad ofrece un camino para superar la fragmentación del saber, aspecto que se ha potenciado por la especialización. Desde esta perspectiva, la interdisciplinariedad permitiría realizar una cierta unidad del saber, no como una reducción a la disciplina, sino como toma de conciencia de la complejidad de las realidades que nos rodean. (Casanova, 2018)

Esto coincide con lo expresado por Fernández-Batanero (2004), quien sostiene que la transversalidad, puede ser vista como una estrategia curricular en el ámbito universitario la cual puede contribuir a establecer puentes de unión entre el saber académico (aprender-aprender) y el saber vital (aprender a vivir). También reconoce que la transversalidad permite afrontar el 
estudio de la realidad de un modo global, en este punto la interdisciplinariedad tiene protagonismo, al emplear las distintas disciplinas como herramientas instrumentales e interpretativas de la realidad, el conocimiento al ser complejo no puede ser visto por parcelas unidisciplinares.

A partir de los planteamientos expresados, se hace indispensable diferenciar lo que se denominara como: áreas, ejes y líneas curriculares, para hilvanar el recorrido del trabajo sin diluirnos en interpretaciones particulares. Dicho lo anterior, se entenderá por líneas curriculares aquellas que se extenderán a lo largo del plan de estudios, su organización diacrónica fija el curso temporal de las unidades curriculares agrupándose de acuerdo con su naturaleza. Las áreas por su lado constituyen espacios intermedios que norman estructuras de relación y profundidad donde en algunos casos podrán contener más de una línea curricular.

Con respecto a los ejes transversales o curriculares (ordenamiento transversal) se observará el entrecruzamiento de líneas y áreas curriculares lo cual determina el espacio de acción donde los conocimientos se solapan y se concatenan para resultar en un aprendizaje complejo. (Casanova, 2018)

En el Modelo de articulación de la estructura curricular planteado por Casanova (2011) los ejes transversales parten desde el macrocurrículo, el número de ejes transversales dependerán de la naturaleza de la profesión y provendrán del análisis consensuado de los actores involucrados en el proceso de diseño del currículo, los mismos serán declarados a nivel de la estructura mesocurricular. Cada eje transversal tocará en más de un punto el nivel micro del currículo de acuerdo con el número de unidades curriculares involucradas en el alcance del eje; en este punto es bueno señalar que una unidad curricular puede converger en varios ejes transversales por su naturaleza compleja e interdisciplinaria.

En este sentido, el Modelo de articulación de la estructura curricular, tiene su asiento en una visión de la interdisciplinariedad que proyecta un tipo de relación horizontal y vertical entre las diferentes áreas del conocimiento, lo cual favorece no sólo a la adquisición de información relevante y significativa, sino también al desarrollo de estructuras de pensamiento y de acción, todo ello sin desconocer la naturaleza de las disciplinas. (Casanova, 2018)

Es importante señalar que este modelo de articulación asume también la posición de Paredes (2011, pág. 180), quien ofrece una visión de la formación integral desde el enfoque por competencias bastante completa, la autora plantea, para:

(...) alcanzar la integralidad desde el enfoque por competencias involucra una renovada forma de pensar los conceptos clave que constituyen la concepción educativa, así, formación integral, competencias, docentes, alumno e institución han de ser asumidos desde una visión compleja que permita la confluencia armónica de elementos y características que garanticen la integralidad de la formación y el compromiso de los actores del currículo en todos los procesos del desarrollo curricular. Desde esta perspectiva, la formación profesional integral ha de considerar dimensiones como la intelectual, humana, social y profesional en la configuración del currículo, permitiendo con ello la transcendencia del hombre por medio del desarrollo de competencias que le permitan una actuación autónoma y reflexiva como reflejo de la integración de saberes y un desempeño profesional integral consciente, comprometido y en 
correspondencia con la realidad contextual en la cual se inserta como ser multidimensional y en constante proceso de formación.

Se reconoce al trabajar desde esta visión de transversalidad curricular, que es un proceso complejo y se requiere realizar la transposición didáctica de los principios para darle viabilidad y evitar rechazos en los operadores del currículo.

De lo expresado anteriormente, se hace evidente la necesidad de establecer lineamientos que permitan aclarar a los actores educativos la forma de hacer el traslado de los fines educativos a los niveles prácticos del currículo, en vista que se suscitan problemas en estos procesos, cuando la actividad académica no es organizada como un proyecto institucional. Es por ello por lo que este trabajo ha pretendido proponer un núcleo procedimental sobre la base al modelo de articulación de la estructura curricular definido por Casanova (2011) quienes desde un ordenamiento transversal pretenden superar el ordenamiento lineal tradicional.

Para ello se estableció como ruta metodológica un enfoque racionalista de carácter deductivo, lo cual implicó la construcción y explicación de una estructura operativa denominada núcleo procedimental como soporte operativo al modelo teórico integración curricular del perfil por competencias a partir de un ordenamiento transversal.

El núcleo procedimental del modelo cuenta de 7 (siete) fases, la primera de ellas está localizada a nivel del macrocurrículo, implica la codificación del perfil académico-profesional bajo el enfoque por competencias; en la segunda fase se conforman los ejes transversales del currículo; en la fase tres se definen, para cada eje transversal se describen los propósitos y objetivos. En la cuarta fase se realiza la selección previa de los indicadores de logro del perfil académico-profesional bajo el enfoque por competencias, esto corresponde a la contribución de cada eje transversal al perfil.

La quinta y sexta fase están localizadas a nivel del microcurrículo; en la quinta fase se realiza la distinción de los indicadores de logro para ser incorporados a las unidades curriculares, seleccionados previamente para el eje transversal. Seguidamente, en la sexta fase, se validan los indicadores de logro cuando se verifica la posibilidad de que los mismos puedan ser evaluados. Una vez finalizada la fase anterior se procede a comparar los indicadores de logro del eje transversal con los definitivos de las unidades curriculares pertenecientes al eje, esta fase involucra un proceso de retroalimentación.

En la séptima y última fase transcurre a nivel mesocurricular, se realiza la selección definitiva de indicadores para cada eje transversal, este procedimiento tiene como insumo lo generado por la fase seis del núcleo procedimental del modelo teórico. Es importante indicar que esta fase requiere que todas las unidades curriculares hayan culminado su proceso de selección y validación de indicadores de logro de las competencias reflejadas en el perfil académicoprofesional.

\section{Núcleo procedimental del modelo de integración de la estructura curricular}

Fases del núcleo procedimental del modelo de integración de la estructura curricular:

1. Codificación del perfil académico-profesional bajo el enfoque por competencias.

Para la codificación del perfil académico-profesional se recomienda que sea elaborada por los gestores curriculares, con la finalidad que los códigos sean comunes para todas las asignaturas del plan de estudio. Los códigos asignados a los elementos de la competencia 
deben ser simples y de fácil comprensión por todos los actores educativos, se recomienda usar las iniciales y el número asignado a la misma, por ejemplo, para la Competencia General 1 Procedimental 1 sería CG1P1. El uso unificado de estos códigos permitirá constatar de forma rápida los indicadores de logro asumidos para cada competencia. No obstante, se ratifica, que la escogencia del tipo de codificación es flexible para cada institución, pero una vez seleccionada debe ser uniforme para todos los niveles curriculares.

Tabla 1. Codificación de Competencias e Indicadores de Logro

\begin{tabular}{|c|c|c|c|c|c|c|c|}
\hline \multirow{2}{*}{\multicolumn{2}{|c|}{ Competencia }} & \multicolumn{6}{|c|}{ Indicadores de logro } \\
\hline & & \multicolumn{2}{|c|}{ Cognoscitivo } & \multicolumn{2}{|c|}{ Procedimental } & \multicolumn{2}{|c|}{ Actitudinal } \\
\hline \multicolumn{8}{|c|}{ Generales } \\
\hline \multirow{2}{*}{1} & \multirow{2}{*}{ CG1 } & 1 & CG1C1 & 1 & CG1P1 & 1 & CG1A1 \\
\hline & & 2 & CG1C2 & 2 & CG1P2 & 2 & CG1A2 \\
\hline \multirow{2}{*}{2} & \multirow{2}{*}{ CG2 } & 1 & CG2C1 & 1 & CG2P1 & 1 & CG2A1 \\
\hline & & & & 2 & CG2P2 & 2 & CG2A2 \\
\hline \multirow{3}{*}{3} & \multirow{3}{*}{ CG3 } & 1 & CG3C1 & 1 & CG3P1 & 1 & CG3A1 \\
\hline & & 2 & CG3C2 & 2 & CG3P2 & 2 & CG3A2 \\
\hline & & & & 3 & CG3P3 & 3 & CG3A3 \\
\hline \multicolumn{8}{|c|}{ Básicas } \\
\hline \multirow{2}{*}{1} & \multirow{2}{*}{ CB1 } & 1 & CB1C1 & 1 & CB1P1 & 1 & CB1A1 \\
\hline & & 2 & CB1C2 & 2 & CB1P2 & 2 & CB1A2 \\
\hline \multicolumn{8}{|c|}{ Específicas } \\
\hline \multirow{3}{*}{1} & \multirow{3}{*}{ CE1 } & 1 & CE1C1 & 1 & CE1P1 & 1 & CB3A1 \\
\hline & & 2 & CE1C2 & 2 & CE1P2 & 2 & CB3A2 \\
\hline & & 3 & CE1C3 & & & 3 & CB3A3 \\
\hline
\end{tabular}

Fuente: Casanova (2011)

En la Tabla 1 se muestra cómo se van configurando los códigos, esto es un paso de gran importancia ya que de esta matriz se desprenderá el correcto traslado de los indicadores de logro a las unidades curriculares. Otra de las ventajas de esta matriz es que a partir de ella los gestores curriculares elaborarán el plan de seguimiento y podrán evidenciar la coherencia teórica del currículo.

2. Conformación de los ejes transversales asumidos en el currículo

La introducción de la transversalidad en el currículo constituye un cambio de paradigma, que permite, la construcción de un pensamiento crítico, producto de la interacción de diferentes áreas curriculares. Por otra parte, amplía el escenario más allá de los contenidos académicos clásicos, al incorporar oportunamente temas de la realidad cotidiana y problemas actuales de la humanidad, aun no incluidos en las áreas disciplinarias. En la elaboración de los ejes transversales se sugieren las siguientes premisas en la organización y selección, a saber:

- Se observarán las competencias generales asumidas por la institución y las características propias de la carrera o programa. 
- En los procesos de transformación curricular se tomarán en cuenta los ejes transversales vigentes para la fecha de su evaluación.

- Se podrán asumir ejes transversales para una competencia.

- Se recomienda propiciar la coexistencia de varias competencias en un eje transversal cuyo carácter interdisciplinario induzcan el logro de aprendizajes complejos, con la finalidad de fortalecer el perfil de académico profesional propuesto.

3. Definición, propósitos, objetivos del eje transversal

Para la elaboración de la definición, propósitos y objetivos del eje transversal se debe considerar la contribución que hará el mismo a la consecución del perfil académico-profesional. Estos representan una guía para el traslado de los fines educativos, a las unidades curriculares respetando su naturaleza y especificidad.

4. Selección previa para el eje transversal de los indicadores de logro del perfil académicoprofesional bajo en enfoque por competencias.

Se tomarán como guía los elementos desarrollados en la fase anterior, se seleccionarán aquellos indicadores cognoscitivos, procedimentales y actitudinales de las competencias declaradas en el perfil académico profesional. Este procedimiento tiene por finalidad, develar el alcance que tendrá el eje transversal y su contribución para el logro de los fines educativos. El diseño curricular contará con una tabla donde se establezca la contribución de cada eje transversal al perfil académico-profesional, que servirá de referencia para este proceso.

Aun cuando, no se pretende dar una visión global sobre el nivel microcurricular, si se establecerán algunas precisiones en vista que el modelo en su núcleo procedimental requiere de procesos que se desarrollan a este nivel curricular, para la conexión de las disciplinas involucradas en cada eje transversal, se deben considerar los problemas sociales, éticos y morales presentes en su entorno social (pertinencia del conocimiento).

Desde esta perspectiva, el enfoque transversal reconoce la importancia de las disciplinas, para el abordaje de aprendizajes complejos; sin embargo, hace un llamado para la revisión de las estrategias didácticas aplicadas con la finalidad de lograr la interdisciplinariedad. La transversalidad, va más allá de establecer unidades curriculares con contenidos paralelos, sino como medios que conducen a un aprendizaje que propicie la formación científicotecnológica, humanística y ético - moral, de un ser humano cónsono con los cambios sociales y culturales que se suscitan en el mundo actual.

Es importante resaltar que la interdisciplinariedad tiene que ver con la generación de conocimientos en las disciplinas y su integración con otras. Siendo esta la premisa, la selección y organización de los contenidos toma como base no sólo los conocimientos propios de la disciplina, sino su vinculación con un proceso de aprendizaje complejo, donde se deben tomar en cuenta experiencias y actitudes previas de manera tal que un mismo concepto sea abordado desde diversas perspectivas (Casanova, 2018). 
Tabla 2. Listado de Competencias Generales, Básicas y

Específicas abordadas por Eje Transversal

Competencias Generales Indicadores de logro

Competencias Básicas Indicadores de logro

Competencias Específicas Indicadores de logro

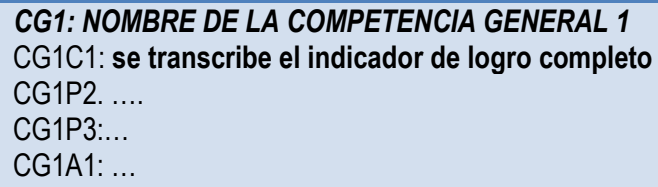

Este procedimiento se realizará para cada competencia asumida por el ejes

Fuente: Casanova (2011)

Las fases cinco y seis del núcleo procedimental del modelo, se ejecutan a nivel del microcurrículo, específicamente en el proceso de planificación de la instrucción de cada unidad curricular. Siendo el diseño instruccional parte de un todo, debe ser coherente con los niveles precedentes del currículo, la concepción educativa asumida debe verse reflejada en este nivel, es indispensable que cada unidad curricular explicite los elementos del perfil que ha decidido arrogarse y evaluar. 
Tabla 3. Listado de Competencias Generales, Básicas y

Específicas abordadas por unidades curriculares.

\begin{tabular}{|c|c|c|}
\hline & Competencias & Eje transversal \\
\hline \multirow{5}{*}{$\begin{array}{l}\text { Competencias Generales } \\
\text { Indicadores de logro }\end{array}$} & \multicolumn{2}{|c|}{ CG1: NOMBRE DE LA COMPETENCIA GENERAL 1} \\
\hline & $\begin{array}{l}\text { CG1C1: se transcribe el indicador } \\
\text { de logro completo }\end{array}$ & ET1, ET2 \\
\hline & CG1P2.... & ET1 \\
\hline & CG1P3:... & ET1 \\
\hline & CG1A1: ... & ET1, ET3 \\
\hline \multirow{4}{*}{$\begin{array}{l}\text { Competencias Básicas } \\
\text { Indicadores de logro }\end{array}$} & \multicolumn{2}{|c|}{ CB1: NOMBRE DE LA COMPETENCIA BASICA 1} \\
\hline & CB1C3:.. & ET1 \\
\hline & CB1P2:... & ET1 \\
\hline & $\begin{array}{l}\text { CE1: NOMBRE DE LA } \\
\text { COMPETENCIA ESPECIFICA } 1\end{array}$ & \\
\hline \multirow{5}{*}{$\begin{array}{l}\text { Competencias } \\
\text { Específicas } \\
\text { Indicadores de logro }\end{array}$} & CE1C3:... & ET1, ET4 \\
\hline & CE1C4:... & ET1 \\
\hline & CE1P1..... & ET1 \\
\hline & CE1A1:... & ET1, ET2 \\
\hline & \multicolumn{2}{|c|}{$\begin{array}{l}\text { Este procedimiento se realizará para cada competencia asumida } \\
\text { por el eje }\end{array}$} \\
\hline
\end{tabular}

Fuente: Casanova (2011)

5. Distinción de los indicadores de logro seleccionados previamente por el eje transversal para ser incorporado a las unidades curriculares.

A diferencia de la selección para cada eje transversal, las unidades curriculares deben partir de los indicadores de logro ya seleccionados previamente por el eje o ejes que asume. Un modelo de matriz para el listado de competencias generales, básicas y específicas abordadas por la unidad curricular se explicita en la Tabla 3, la misma cuenta con una columna adicional donde se indica el eje transversal que refleja el indicador que deriva del perfil académico profesional.

Para llevar a cabo este proceso se hace indispensable que cada eje transversal se encuentre también codificado, esta será una función que debe ser adjudicarse a la comisión de currículo de la institución para evitar múltiples codificaciones que eviten un proceso unificado en el plan de estudio considerado.

6. Validación en los diseños instruccionales de los indicadores de logro a asumir por las unidades curriculares donde se verifica la posibilidad de ser evaluados.

Esta fase se desarrolla en el proceso organización y sistematización de las unidades temáticas de los diseños instruccionales de las unidades curriculares. En el desarrollo de la secuencia instruccional, se contemplan los elementos que permiten planificar el proceso de acción didáctica a desarrollarse en los escenarios de aprendizaje, lo cual permite verificar la 
posibilidad de evaluación de los indicadores de logro, para ello se toma en cuenta su naturaleza de la unidad curricular.

Siendo que cada área del saber cuenta con particularidades en el desarrollo de su proceso formativo, se sugiere que los formatos para la planificación de las secuencias didácticas sean de fácil comprensión, adaptados a las necesidades y naturaleza de la unidad curricular, en las Tablas 4, 5 y 6 se presentan tres formatos que permiten la organización y sistematización de las unidades temáticas.

En la Tabla 4 se describe que debe contener cada una de las columnas que será similar para las demás tablas, en el caso de los formatos presentados en las Tablas 5 y 6 las unidades curriculares cuentan con un tiempo asignado por semanas que no varía lo que facilita la planeación y la comprensión por parte de los actores educativos.

Tabla 4. Desarrollo de la secuencia de aprendizaje. Modelo para unidades curriculares teóricas

\begin{tabular}{|c|c|c|c|c|c|}
\hline \multicolumn{2}{|c|}{ UNIDAD 1} & \multicolumn{4}{|c|}{ OBJETIVOS TERMINALES } \\
\hline $\begin{array}{l}\text { Objetivos } \\
\text { didácticos }\end{array}$ & $\begin{array}{c}\text { Contenido } \\
\text { Programático }\end{array}$ & $\begin{array}{l}\text { Estrategias y } \\
\text { Recursos } \\
\text { Instruccionales }\end{array}$ & $\begin{array}{l}\text { Estrategias de } \\
\text { Evaluación } \\
\text { IEv: Indicadores } \\
\text { Evaluados }\end{array}$ & Acreditación & Tiempo \\
\hline $\begin{array}{l}\text { Logros } \\
\text { específicos de } \\
\text { la unidad o } \\
\text { tema, se debe } \\
\text { contemplar los } \\
\text { indicadores de } \\
\text { logro a evaluar }\end{array}$ & $\begin{array}{l}\text { Se listan los } \\
\text { contenidos y se } \\
\text { organizan de } \\
\text { acuerdo a los } \\
\text { temas } \\
\text { considerados } \\
\text { para la unidad. } \\
\text { Se deja abierta } \\
\text { la posibilidad de } \\
\text { separar el mismo } \\
\text { en las diferentes } \\
\text { dimensiones del } \\
\text { saber }\end{array}$ & $\begin{array}{l}\text { Acción didáctica y } \\
\text { todos los medios a } \\
\text { ser abordados de } \\
\text { acuerdo a la } \\
\text { temática y la } \\
\text { estrategia de } \\
\text { evaluación } \\
\text { seleccionada. }\end{array}$ & $\begin{array}{l}\text { Se selecciona la } \\
\text { estrategia más } \\
\text { adecuada que permita } \\
\text { la evaluación de los } \\
\text { indicadores de logro. } \\
\text { Se debe contemplar la } \\
\text { dimensión del saber } \\
\text { que se espera } \\
\text { alcanzar. } \\
\text { Se utilizan sólo los } \\
\text { códigos para } \\
\text { identificar los } \\
\text { indicadores de logro } \\
\text { evaluados para cada } \\
\text { tema. }\end{array}$ & $\begin{array}{l}\text { Se indica la } \\
\text { ponderación que } \\
\text { tendrá cada } \\
\text { tema o } \\
\text { evaluación de la } \\
\text { unidad } \\
\text { correspondiente }\end{array}$ & $\begin{array}{l}\text { Se señala el } \\
\text { tiempo del } \\
\text { proceso } \\
\text { instruccional }\end{array}$ \\
\hline
\end{tabular}

Fuente: Casanova (2011) 
Tabla 5. Desarrollo de la secuencia de aprendizaje. Modelo para unidades curriculares teóricas-prácticas

\begin{tabular}{|c|c|}
\hline UNIDAD & OBJETIVOS TERMINALES \\
\hline UNIDAD I & SEMANA 1 \\
\hline Objetivos específicos & \\
\hline Unidades temáticas & \\
\hline Estrategias de aprendizaje \\
Actividad teórica
\end{tabular}

Fuente: Elaboración propia

Tabla 6. Desarrollo de la secuencia de aprendizaje. Modelo para unidades curriculares clínicas

UNIDAD CURRICULAR

\section{OBJETIVOS TERMINALES}

\begin{tabular}{|c|c|c|}
\hline ETAPA & Semana 1 & Semana 2 \\
\hline Objetivos didácticos & & \\
\hline Contenidos & & \\
\hline Estrategias de aprendizaje & \\
\hline Recursos instruccionales & \\
\hline Estrategias de Evaluación & \\
\hline Indicadores evaluados & \\
\hline
\end{tabular}

Fuente: Elaboración propia

Como se observa, en las tablas antes presentadas para esta fase, se hace una propuesta para la sistematización de las unidades temáticas en los diseños instruccionales donde se le da especial importancia a la declaración de los indicadores de logro de cada competencia en el proceso de evaluación por cuanto se considera un elemento fundamental en 
los currículos que adoptan el enfoque por competencias; por supuesto se considera una propuesta que permite visualizar el desarrollo de la secuencia de aprendizaje, se deja abierta la posibilidad de profundizar en la construcción de metodologías que aborden esta fase del proceso. Retroalimentación y selección definitiva de los indicadores de logro del perfil académico-profesional bajo para el eje transversal en el enfoque por competencias.

El proceso de planificación de la instrucción involucra, la definición de las unidades temáticas, cada una de ellas, contará con un listado de los indicadores que serán evaluados; asimismo, se establecen los objetivos terminales que hace referencia al alcance de la unidad correspondiente. Un elemento que se debe considerar es el uso de objetivos didácticos, ellos permitirían integrar indicadores y contenidos propios de la disciplina, asimismo orientan la experiencia didáctica.

En esta fase se regresa de nuevo al nivel mesocurricular, y se da inicio, una vez concluidos la construcción de los diseños instruccionales del diseño curricular. En ese momento, se procede a realizar una contrastación de los indicadores con la finalidad de realizar la selección definitiva de los indicadores de logro del perfil académico profesional. Se recomienda que cada una de las unidades curriculares introduzca una tabla (Tabla 7) donde se visualice su contribución al perfil, con la finalidad de simplificar el proceso de gestión curricular.

Tabla 7. Cuadro resumen de las competencias abordadas por la unidad curricular

\begin{tabular}{|l|c|c|c|}
\hline \multicolumn{1}{|c|}{ COMPETENCIAS } & \multicolumn{3}{c|}{ INDICADORES DE LOGRO } \\
\hline & Cognitivo & Procedimental & Actitudinal \\
\hline Nombre de la competencia general & Se coloca sólo el número del indicador de logro \\
\hline Nombre de la competencia general & $1,2,3$ & 2,4 & 1 \\
\hline $\begin{array}{l}\text { Competencias básicas } \\
\text { Nombre de la competencia básica }\end{array}$ & & & \\
\hline $\begin{array}{l}\text { Competencias específicas } \\
\text { Nombre de la competencia } \\
\text { específica }\end{array}$ & & & \\
\hline
\end{tabular}

Fuente: Casanova (2011)

Se sugiere a los gestores curriculares, construir una matriz donde se lleve el consolidado de las competencias abordadas en el plan de estudio (Tabla 8), la misma con la ayuda de la codificación elaborada debe reflejar los indicadores de logro abordados por cada unidad curricular, lo cual permite verificar si se contemplan la totalidad del perfil académicoprocedimental desde las unidades curriculares. Esto no debe confundirse con un proceso de certificación de competencias por cuanto el mismo sólo puede ser evaluado en los futuros profesionales. 
Tabla 8. Cuadro resumen de las competencias abordadas en el plan de estudio

\begin{tabular}{|c|c|c|c|c|c|}
\hline AREA & UNIDADES & EJE & \multicolumn{3}{|c|}{ COMPETENCIAS } \\
\hline CURRICULAR & CURRICULARES & TRANSVERSAL & Generales & Básicas & Especificas \\
\hline & & & & & \\
\hline & & & & & \\
\hline & & & & \\
\hline
\end{tabular}

Fuente: Casanova (2011)

Uno de los elementos de importancia de este estudio se centra en el rol que se le otorga a la gestión académica del currículo, aspecto que devela el carácter protagónico del nivel mesocurrícular que ha sido trabajado escasamente en el desarrollo de los diseños curriculares, lo que ha impedido la articulación de la estructura curricular y por ende la posibilidad de inconsistencias en el sistema curricular.

Siendo que las instituciones educativas independientemente de su nivel en el sistema de educación han evolucionado como organizaciones complejas, dispuestas al mejoramiento continuo de sus procesos con la finalidad de lograr estándares de calidad, pero con pertinencia social; se hace imprescindible, desde la mirada de las diferentes disciplinas que alimentan el proceso de gestión académica, adaptar sus técnicas al hecho educativo, sin dejar de recordar que su tangible más valioso es el talento en formación.

La gestión curricular involucra procesos de planificación sostenidos relacionados con el diseño, implementación, desarrollo y evaluación permanente tanto de las fases anteriores como del currículo. Lo anterior implica procesos de monitoreo permanente de su operatividad, con la finalidad de acercar en el currículo lo formal escrito, a lo real ejecutado. Asimismo, la gestión curricular involucra entre otras cosas la definición de políticas y la caracterización de estructuras académicas según nivel educativo, tomar en cuenta las previsiones para el funcionamiento y la organización de los recursos.

La gestión curricular maneja los mismos principios que son extrapolados de la gestión de organizaciones productivas por supuesto se hacen las adaptaciones correspondientes para el proceso educativo, algunos de los que se pueden citar son: gestión sistémica, proactiva, flexible y estratégica, audaz, abierta al aprendizaje permanente, asertiva, integradora y descentralizadora.

Es importante entender que el currículo es un plan, que se desarrolla de forma continua en todos sus niveles (macro, meso y microcurrículo), es por ello por lo que se hace necesario acercarnos a su definición; para Ander-Egg (2007), la planificación es la acción consistente en utilizar un conjunto de procedimientos mediante los cuales se introduce una mayor racionalidad y organización en un conjunto de actividades articuladas entre sí, las mismas deben ser previstas anticipadamente.

Por otra parte, el proceso de gestión curricular parte del diagnóstico de las necesidades, para ello requiere visualizar las características tanto de la institución, como las de su entorno y nivel educativo, con el propósito de tomar decisiones que propendan a la ejecución de acciones 
encaminadas garantizar el logro de los objetivos propuestos a medida que mejorar la calidad de la educación.

La planificación aporta beneficios al proceso de gestión curricular, por cuanto genera mayores niveles de compromisos de los actores con los procesos que llevan a cabo en su respectivo nivel, mientras sea socializado y sean involucrados en su construcción. Asimismo, abre múltiples posibilidades de acción y posibilita la relación entre las metas institucionales y el mejoramiento de los procesos educativos. Por otro lado, posibilita la relación entre lo académico, lo comunitario, lo administrativo- financiero, lo pedagógico-curricular; permite una mayor relación entre la organización y la sociedad la cual se posiciona como una fuente de aprendizaje y desarrollo de la identidad cultural de los educandos.

Si se emprenden procesos de gestión eficientes, estos deben estar acompañados de manera indisoluble con la evaluación. Por su lado Díaz-Barriga (2005) refiere que la evaluación curricular, analiza los principales contrastes, continuidades y rupturas en materia curricular. Vílchez (2005) lo define como un proceso participativo donde se delinea, obtiene y analiza información útil para contrastarla contra un patrón, con el propósito de juzgar y tomar decisiones alternativas respecto de la concepción, estructura, funcionamiento y resultados del currículo.

Por su parte Fernández (2004) refiere que la evaluación curricular presenta una diversidad de enfoques; transita desde concepciones que asumen la evaluación como emisión de juicios de valor, pasando por aquellas que centran su importancia en la toma de decisiones, hasta las que enfatizan los aspectos éticos. La evaluación tiende así al servicio de los valores públicos y de los justos intereses de los actores involucrados en el quehacer educativo.

En este sentido la evaluación debe ser asumida, como un proceso continuo de indagación de información con el objeto de contrastarla con los objetivos propuestos y realimentar el currículo; se debe evaluar el plan (documento), los recursos (infraestructura, financieros, instruccionales, entre otros) y a todos los actores del hecho educativo. Asimismo, este proceso deberá ser abordado en el diseño, desarrollo, implementación y también en la evaluación del currículo.

Es indiscutible que una gestión adecuada del currículo juega un rol protagónico en la calidad y logro de los fines educativos, por tanto, en todos los procesos asumidos por la institución. Hoy, cada institución debe contar con procesos de planificación y autoevaluación sostenidos en el tiempo, si buscan fortalecer todas sus funciones para ofrecer un servicio de calidad, esta nueva visión de gestionar requiere que las instituciones aprendan, lo que provoca un impacto inmediato en su contexto, por cuanto ellas buscarán cada vez más, ser pertinentes a las necesidades de su entorno.

\section{Conclusiones}

A continuación, algunos elementos que es necesario retomar a manera de cierre:

La educación debe reflejar la concepción de hombre, fortalecer los valores y la cultura; asimismo debe ser integral y para toda la vida e ir más allá de aspectos meramente científicotecnológicos de una profesión incorporando la formación humana, esto lo valida el trabajo emprendido por Paredes (2011).

Es importante propiciar la unificación de criterios sobre las definiciones que serán abordadas por la institución, para este trabajo se asumieron los conceptos surgidos del trabajo 
realizado por Casanova (2011), quien propone considerar para los niveles curriculares los siguientes aspectos:

- Nivel macro: donde se expresa de forma prescriptiva la concepción de hombre, de educación y sus propósitos, cuya expresión resumida será el perfil académico-profesional. - Nivel meso: representado por la malla curricular, explicitándose el alcance de las áreas, ejes curriculares (organización temporal) y ejes transversales (organización lógica del conocimiento), así como las previsiones de gestión curricular.

- $\quad$ Nivel micro: se considera como el plano de los programas de las unidades curriculares.

Por otro lado, en el proceso educativo se reconoce que los siguientes aspectos son oportunidades, aun cuando se pretende que sean incorporados de manera definitiva como componentes del currículo para ser transformados en fortalezas y potenciar la formación profesional:

- Formación integral

- Elaboración de los currículos por competencias

- Educación apoyada en el principio de interdisciplinariedad

- Reconocimiento de las disciplinas

- La transversalidad

Finalmente, se hace imprescindible recapitular en la importancia de visualizar la transversalidad como componente del currículo, que permite su organización y coherencia; al mismo tiempo que ofrece los mecanismos operativos para recorrerlo tanto en forma diacrónica como sincrónica. Adicional a esto, a través de la transversalidad se profundiza y consolidan todas las fases del desarrollo curricular además que se fortalece la formación integral, al proyectar el trabajo curricular en sus diversos niveles de concreción, pero sobre todo le da fuerza al nivel mesocurricular como soporte de la integración de la estructura, de esta manera se facilita el desarrollo de competencias académico-profesionales, tomando previsiones para la evaluación de sus indicadores de logro.

\section{Referencias bibliográficas}

Ander-Egg, Ezequiel. (2007). Introducción a la planificación estratégica. Grupo Editorial Lumen. Argentina.

Bravo, Esperanza. (2006). La transversalidad como vía para la formación integral. Tesis Doctoral, Doctorado en Ciencias Humanas, Universidad del Zulia, Venezuela.

Bustamante, Suleima y Sánchez, José. (2007). Reforma, cultura democrática y transversalidad "Implicaciones en la relación universidad-sociedad". Revista TELOS. Volumen 9, No.

2. Venezuela (Pp. 207--220)

Casanova, llya. (2011). Transversalidad y Desarrollo de Competencias Profesionales. Tesis

Doctoral, Doctorado en Ciencias Humanas. Universidad del Zulia. Venezuela.

Casanova, llya. (2018). Conexión de la estructura curricular desde la transversalidad. En Paredes Ítala, Casanova llya y Naranjo Miguel, Formación integral, enfoque por competencias y transversalidad curricular en la educación superior. Editorial UTN. Ecuador.

Coll, Cesar. (2001). Fundamentos del currículum. Currículum de Postgrados: Aspectos Conceptuales y Prácticos. Diálogos Universitarios de Postgrado. Perú. 
Díaz-Barriga, Frida. (2005). Desarrollo del currículo e innovación; Modelos e investigación en los noventa. Revista Perfiles educativos. Volumen 27 Número 107, México, (Pp. 57-84)..

Fernández, Norberto. (2004). Hacia la convergencia de los sistemas de educación superior en América Latina. Revista Iberoamericana de Educación, Número 35. (Pp. 39-71). Retrieved from www.rieoei.org/rie35a02.htm

Fernández-Batanero, José. (2004). La transversalidad curricular en el contexto universitario: un puente entre el aprendizaje académico y el natural. Revista Fuentes, Número 5. Retrieved from https://revistascientificas.us.es/index.php/fuentes/article/view/2403 Horruitiner, Pedro. (2006). El reto de la transformación curricular. Revista Iberoamericana de Educación, 40(3). Retrieved from http://www.rieoei.org/deloslectores/1524Silva.pdf

Martín-Barbero, Jesús. (2003). Saberes hoy: diseminaciones, competencias y transversalidades. Madrid: Revista Iberoamericana de Educación. (Vol. 32). Retrieved from http://www.rieoei.org/rie32a01.htm

Paredes, İtala. (2011). Formación Profesional integral desde el enfoque por competencias. Tesis Doctoral, Doctorado en Ciencias Humanas, Universidad del Zulia, Venezuela.

Peñaloza, Walter. (2005). Tecnología Educativa (tercera edición). Escuela Empresarial Andina del Convenio Andrés Bello. Perú.

Vílchez, Nerio Gerardo. (2004). Una revisión y actualización del concepto de Currículo. Revista TELOS. Volumen 6, No. 2. Venezuela (Pp. 194-208).

Vílchez, Nerio Gerardo. (2005). Fundamentos del currículo. Fondo Editorial Urbe. Venezuela. 